\title{
Cirurgia laparoscópica na apendicite aguda em grávidas: revisão bibliográfica
}

\author{
Laparoscopic surgery in cases of acute appendicitis in pregnant patients: \\ bibliographic review
}

Cirugía laparoscópica en casos de apendicitis aguda en pacientes embarazadas:
revisión bibliográfica

Larissa Silveira Batista ${ }^{1 *}$, Bárbara Franco Dourado², David Pereira Bezerra ${ }^{3}$, Emanuelle Ferrão Lopes $^{4}$, Gabriel Lima Brandão Monteiro ${ }^{5}$, Jeanne Christini da Silva Rosa ${ }^{6}$, Talita Vitória Vieira Abdo ${ }^{7}$, Wagner Pablo Corrêa ${ }^{8}$.

\section{RESUMO}

Objetivo: Explorar na literatura acerca do uso da videolaparoscopia (VLP) como intervenção no tratamento da apendicite aguda em gestantes e as possíveis implicações desse procedimento na saúde materna-fetal. Revisão bibliográfica: Verificou-se a predominância de vantagens na abordagem laparoscópica em comparação à apendicectomia aberta, uma vez que configura-se um método seguro e minimamente invasivo. Sendo uma técnica mais difundida atualmente, dados de pesquisas mais recentes sugerem que a técnica laparoscópica possui menor probabilidade de complicações, além de funcionar como um método não só diagnóstico como também terapêutico, que pode ser realizado em qualquer trimestre da gravidez. Algumas complicações como lesão ao útero gravídico, diminuição do fluxo sanguíneo uterino e eventos tromboembólicos para a gestante, bem como menor risco de depressão respiratória para o feto, foram evidenciadas. As diferenças entre as técnicas foram apresentadas, revelando um menor risco de hospitalização, infecções, irritação uterina, hemorragias, dor pós-operatória e íleo paralítico na cirurgia laparoscópica. Considerações finais: A abordagem laparoscópica é mais segura que a apendicectomia aberta e deve ser considerada como procedimento padrão em pacientes grávidas.

Palavras-chave: Apendicite, Gravidez, Laparoscopia, Apendicectomia.

\begin{abstract}
Objective: Explore in the literature the use of the video laparoscopic (VLP) approach as intervention for treatment of acute appendicitis in pregnant patients and possible implications of the procedure in fetal-maternal health. Bibliographic review: It has been verified the prevalence of advantages of the laparoscopic approach over open appendectomy, once this method is safer and minimally invasive. Since the technique is more widespread nowadays, data from more recent research suggests that the laparoscopic technique has a lower probability to cause complications to the pregnant patient and the fetus, in addition to working not only as a diagnostic but also therapeutic method, that can be realized in any trimester of pregnancy. Some complications such as lesions to the pregnant uterus, decrease of uterine blood flow and less thromboembolic events for the pregnant patient, as well as lesser risk of respiratory depression for the fetus, were evidenced. Differences between the techniques were presented, revealing a lesser risk of hospitalization, infections, uterine irritation, hemorrhages, postoperative pain and paralytic ileus in the laparoscopic surgery. Final considerations: The laparoscopic approach is safer than open appendectomy and should be considered as a standard procedure in pregnant patients.
\end{abstract}

Keywords: Appendicitis, Pregnancy, Laparoscopy, Appendectomy.

1 Centro Acadêmico Barão de Mauá (CABM), Ribeirão Preto - SP. *E-mail: larissasbatista@hotmail.com

2 Universidade José do Rosário Vellano (Unifenas), Belo Horizonte - MG.

${ }^{3}$ Faculdade de Medicina Estácio de Juazeiro do Norte (Estácio FMJ), Juazeiro do Norte - CE.

${ }^{4}$ Universidade Católica de Pelotas (UCPel), Pelotas - RS.

${ }^{5}$ Centro Universitário do Planalto Central (Uniceplac), Brasília - DF.

6 Universidade do Sul de Santa Catarina - Pedra Branca (UNISUL PB), Palhoça - SC.

${ }^{7}$ Faculdade Atenas Passos (Uniatenas), Passos - MG.

${ }^{8}$ Faculdade de Medicina do Vale do Aço (Univaço), Ipatinga - MG.

SUBMETIDO EM: 3/2021

ACEITO EM: 3/2021

PUBLICADO EM: 6/2021 


\section{RESUMEN}

Objetivo: Explorar la literatura sobre el uso de la videolaparoscopia (VLP) como intervención en el tratamiento de la apendicitis aguda en mujeres embarazadas y las posibles implicaciones de este procedimiento en la salud materno-fetal. Revisión bibliográfica: Hubo un predominio de ventajas en el abordaje laparoscópico en comparación con la apendicectomía abierta, ya que es un método seguro y mínimamente invasivo. Al tratarse de una técnica que está más extendida en este momento, datos de investigaciones más recientes sugieren que la técnica laparoscópica tiene menos probabilidades de tener complicaciones, además de funcionar como método diagnóstico así como terapéutico, que puede realizarse en cualquier trimestre de embarazo. Se evidenciaron algunas complicaciones como lesión del útero gestante, disminución del flujo sanguíneo uterino y eventos tromboembólicos para la gestante, así como un menor riesgo de depresión respiratoria para el feto. Se presentaron las diferencias entre las técnicas, revelando un menor riesgo de hospitalización, infecciones, irritación uterina, hemorragias, dolor postoperatorio e íleon paralítico en la cirugía laparoscópica. Consideraciones finales: El abordaje laparoscópico es más seguro que la apendicectomía abierta y debe considerarse como un procedimiento estándar en pacientes embarazadas.

Palabras clave: Apendicitis, Embarazo, Laparoscopia, Apendicectomía.

\section{INTRODUÇÃO}

Durante a gestação, o organismo materno passa por diversas adaptações fisiológicas, podendo desenvolver doenças também comuns em mulheres não grávidas como, por exemplo, a apendicite aguda (FIGUEIREDO FAS e CORSO CO, 2002). A apendicite apresenta-se como uma emergência e é a causa mais comum de abdome agudo e de cirurgias não obstétricas em gestantes, ocorrendo em cerca de 1:500 a 1:635 gestações por ano, sendo mais frequente no segundo trimestre (FRANCA NETO AH, et al., 2015). Já a perfuração do apêndice ocorre na maioria das vezes durante o terceiro trimestre de gestação (REEDY MB, et al., 1997).

Na literatura é possível encontrar a descrição de taxas elevadas de morbimortalidade materno-fetais decorrentes da apendicite durante a gravidez. Estas morbimortalidades podem ser explicadas pelo atraso no diagnóstico, uma vez que as modificações fisiológicas características da gestante são muito semelhantes aos sintomas presentes na apendicite, como a ocorrência de vômitos, desconforto abdominal e leucocitose discreta (DALAQUA M e CORSI PR, 2006).

Além da apresentação clínica similar entre sintomas da gestação e aqueles pertencentes a apendicite, um outro fator que pode dificultar o diagnóstico preciso é a presença do feto, visto que pode limitar a utilização de exames de imagem convencionais, como a tomografia computadorizada, um exame geralmente contraindicado por causa dos riscos da exposição fetal à radiação ionizante (CHAKRABORTY J, et al., 2019; IBIEBELE I, et al., 2018). Desta forma, a ultrassonografia se apresenta como a melhor opção para o diagnóstico por imagem da apendicite aguda e deve ser a primeira escolha, seguida pela ressonância magnética; apesar de serem métodos que podem sofrer limitações pelas alterações anatômicas da gestante (IBIEBELE I, et al., 2018).

Quando houver uma suspeita clínica de apendicite ou se for possível confirmar o diagnóstico através de exames de imagem, a abordagem cirúrgica deve ser realizada o mais precocemente possível (DALAQUA M e CORSI PR, 2006). Uma das contraindicações para a realização de uma apendicectomia laparoscópica é a gravidez, entretanto, quando necessário, tal procedimento tem se mostrado rotineiro e bem-sucedido. É de suma importância expor que há uma segurança de não inferioridade de apendicectomia laparoscópica comparando-se a apendicectomia aberta em relação aos desfechos cirúrgicos não houve diferenças significativas entre abortos espontâneos e risco de prematuridade (CHIRURG, 2019)

Apesar de alguns trabalhos ainda considerarem importante as preocupações com a perda fetal e trabalho de parto prematuro, diversos outros estudos mostraram que a laparoscopia é segura e não apresenta resultados cirúrgicos e obstétricos desfavoráveis em relação à técnica aberta (CHAKRABORTY J, et al., 2019; FROUNTZAS M, et al., 2019). Atualmente, torna-se cada vez mais comum o emprego da apendicectomia videolaparoscópica (VLP), já que esta abordagem tem apresentado algumas vantagens em relação à 
apendicectomia aberta (AA), dentre elas, melhor visualização do apêndice pelo cirurgião e melhor avaliação para diagnóstico diferencial. Também há relatos de menor taxa de infecção da ferida pós-operatória, dor de menor intensidade e redução do tempo de internação hospitalar (CHAKRABORTY J, et al., 2019).

O presente trabalho objetiva revisar na literatura sobre o uso da VLP para tratamento da apendicite aguda em pacientes grávidas e as possíveis implicações desse procedimento na saúde materna e fetal.

\section{REVISÃO BIBLIOGRÁFICA}

\section{Sobre a videolaparoscopia}

A apendicectomia como tratamento de escolha para apendicite aguda (AA) é realizada desde 1735 quando foi realizada pela primeira vez, e passou a ser o método de escolha principalmente após a descrição do procedimento feita por McBurney, em 1894. Como a apendicite é uma condição clínica relativamente comum, ao longo do tempo, a apendicectomia passou a ser uma das cirurgias mais realizadas no mundo. Durante mais de um século, a abordagem cirúrgica de uma maneira geral se manteve praticamente sem alterações. No entanto, após o surgimento da cirurgia VLP, no ano de 1983 foi descrita a primeira apendicectomia por videolaparoscopia a qual, mesmo tendo sido pouco aceita no passado, atualmente, esta técnica tem sido amplamente realizada e ainda é objeto de estudos de diversos pesquisadores (JASCHINSKI T, et al., 2018; QUAH G, et al., 2019).

Com base nos estudos de Walker HGM, et al. (2014), tanto a AA quanto a gravidez normal sem complicações podem estar associadas a dor abdominal, anorexia, náuseas e vômitos, aumento da frequência cardíaca e leucocitose. Além disso, o deslocamento anatômico para cima do apêndice pelo útero grávido durante a gravidez pode obscurecer os sinais abdominais clássicos de AA. Como regra geral, a suspeita clínica de apendicite aguda durante a gravidez é indicação de intervenção cirúrgica de urgência, a fim de evitar o desenvolvimento de complicações graves tanto para o feto quanto para a mãe. O diagnóstico por imagem pode ajudar a estabelecer o diagnóstico ou descartar outras causas de dor abdominal aguda na gravidez; no entanto, nem sempre são conclusivos. A abordagem cirúrgica para apendicectomia pode ser aberta (OA) ou laparoscópica (LA).

De acordo com Tinoco-González J, et al. (2018), a apresentação clínica atípica de AA durante a gravidez torna o diagnóstico difícil e pode levar a uma maior incidência de AA complicada e infecção de ferida operatória, bem como a internações mais prolongadas, principalmente quando AA se apresenta no terceiro trimestre.

O diagnóstico preciso e imediato de AA deve ser feito para evitar exploração desnecessária e ter como objetivo a intervenção cirúrgica oportuna na gestante com suspeita de apendicite. A apendicectomia deve ser realizada o mais rápido possível quando a ressonância magnética for sugestiva de apendicite (AGGENBACH L, et al., 2015). Diagnosticar o quadro de apendicite aguda em uma gestante torna-se ainda mais complexo uma vez que os exames laboratoriais sofrem alterações na gestação, como a leucocitose discreta fisiológica já mencionada anteriormente, e os exames com radiação ionizante são contra-indicados devido à possibilidade de indução de malformações fetais. Sendo assim, a ultrassonografia é o exame mais indicado, principalmente no segundo e terceiro trimestres, devido às elevadas taxas de sensibilidade e especificidade (FIGUEIREDO FAS e CORSO CO, 2002).

A dificuldade do diagnóstico clínico ocorre devido à similaridade dos sintomas da apendicite aguda com os sintomas inespecíficos da gravidez, como dor abdominal e vômito, que acabam sendo atribuídos à gestação em curso (DALAQUA M e CORSI PR, et al., 2006).

Sabe-se que no terceiro trimestre de gestação ocorrem alterações no organismo da gestante como, por exemplo, o útero pode deslocar o apêndice cecal para posições variantes dentro da cavidade abdominal, além da ocorrência das contrações de Braxton-Hicks, mais frequentes no final da gestação, que dificultam a formação de aderências e o consequente bloqueio apendicular (FIGUEIREDO FAS e CORSO CO, 2002). 
Devido às alterações anatômicas e fisiológicas inerentes à condição de prenhes, muito se tem discutido sobre a dificuldade do diagnóstico correto no quadro de dor abdominal aguda, não só pelas alterações anatômicas e laboratoriais que por muitas vezes mascaram o quadro, mas também pelo índice de suspeição (FUENTES-RIVAS A, et al., 2011; BARBER-MILLET S, et al., 2016; TOWNSEND CM, et al., 2014).

O útero pode obstruir e inibir o movimento do omento maior para a área de inflamação, modificando ainda mais o quadro clínico. Como consequência do crescimento uterino, o apêndice pode ser deslocado para cima e para o lado direito, assim, no primeiro trimestre a dor se localiza na fossa ilíaca direita em $90 \%$ dos casos, $75 \%$ no segundo trimestre e apenas $37 \%$ no terceiro trimestre (FIGUEIREDO FAS, et al., 2002).

As intervenções cirúrgicas durante a gravidez devem minimizar o risco fetal, sem comprometer a segurança da mãe. No entanto, para obter resultados favoráveis para a gestante e o feto, é necessário um diagnóstico preciso e oportuno, com intervenção imediata (JONATHAN P, et al., 2017).

A laparoscopia é um método seguro e eficaz para o tratamento da apendicite aguda durante a gravidez e tem todos os benefícios de um procedimento minimamente invasivo, mesmo em comparação com mulheres não grávidas. Para obter o melhor resultado, a intervenção deve ser realizada por cirurgiões laparoscópicos experientes (PERRY Z, et al., 2010).

As técnicas que podem ser realizadas para criar um pneumoperitônio na abordagem laparoscópica podem ser divididas em aberta ou fechada. Na técnica fechada, é possível utilizar a agulha de Veress, baseado em um estudo de Lachman E, et al. (1999) onde os autores recomendam dois sítios de punção alternativos: a área subxifóidea e o quadrante superior esquerdo abdominal na linha hemiclavicular $(2 \mathrm{~cm}$ abaixo da caixa torácica). Esses sítios diminuem o risco de perfuração uterina pela agulha. Outra técnica recomendada é o método aberto de Hasson, feito através da passagem de um trocarte após a criação de um pequeno orifício na cavidade abdominal, diminuindo assim, lesões vasculares e viscerais.

No estudo de Ishaq A, et al. (2018), a localização anatômica do apêndice na gravidez foi encontrada em sua posição normal, abaixo ou diretamente na crista ilíaca, em $81.8 \%$ das pacientes, e acima da crista ilíaca em $18.2 \%$ das pacientes. Houve uma média de três centímetros de migração para cima da crista ilíaca, com deslocamento do ponto habitual de McBurney. Identificar corretamente a posição anatômica do apêndice ajuda no posicionamento correto da incisão, no melhor manejo perioperatório e, consequentemente, ajuda no bem-estar fetal e na recuperação da paciente.

A avaliação da frequência cardíaca do feto por ausculta e monitoramento deve ser realizada antes e depois da intervenção, junto com uma avaliação de ultrassom (Manning Score). A maioria dos medicamentos anestésicos atravessam a barreira hemato-placentária e é comum observar alterações na frequência cardíaca fetal ou diminuição da capacidade de resposta fetal. $O$ acesso venoso deve ser estabelecido antes de iniciar qualquer operação e todas as técnicas usadas para controlar o risco de sangramento nas grandes cirurgias são aplicáveis, incluindo a manutenção da normotermia, monitoramento da profundidade da anestesia, e corrigir a hipotensão intra operatória, a fim de limitar as repercussões fetais relacionado a diminuição do fluxo sanguíneo útero-placentário (BOUYOU J, et al., 2015)

\section{A laparoscopia em comparação com a cirurgia aberta}

Quando o exame de imagem não está disponível ou é inconclusivo, o uso da laparoscopia como ferramenta diagnóstica pode ser considerado, porém, ela deve ser usada com cautela, pois pode haver um risco aumentado de parto prematuro e morte fetal após o procedimento para uma apendicite presumida. Além disso, os riscos e benefícios da laparoscopia diagnóstica para outras condições durante a gravidez não tem estado bem documentados e requerem estudos mais amplos (AGGENBACH L, et al., 2015). Os riscos do diagnóstico tardio devem ser considerados em detrimento da realização de uma laparoscopia que não evidencie doença propriamente dita. O cirurgião deve estar preparado para tratar outras possíveis doenças diagnosticadas durante o procedimento. Os benefícios da laparoscopia durante a gravidez têm evidências de serem semelhantes das em pacientes não grávidas, dentre os quais podem ser citados a diminuição do tempo de internação e o retorno de forma mais rápida ao trabalho (FERREIRA PEL, et al., 2020). 
A cirurgia laparoscópica também apresenta vantagens devido à mobilização precoce da paciente, incluindo menos dor e menos ocorrência de íleo paralítico pós-operatório. A abordagem laparoscópica possui ainda outros aspectos vantajosos, incluindo a diminuição da depressão respiratória fetal, devido à diminuição do narcótico pós-operatório, menor risco de complicações da ferida, diminuição da hipoventilação materna pósoperatória, diminuição do risco de eventos de tromboembolismo e menor irritabilidade uterina (FERREIRA PEL, et al., 2020; DALAQUA M e CORSI PR, et al., 2006). A visualização mais definida que a laparoscopia proporciona ao cirurgião pode reduzir a necessidade de manipulação uterina. Dessa forma, a laparoscopia pode ser realizada com segurança durante qualquer trimestre de gravidez uma vez que for indicada.

Apesar disso, tradicionalmente, a recomendação para procedimentos não emergentes durante a gravidez seria evitar a cirurgia durante o primeiro e terceiro trimestres para minimizar o risco de aborto espontâneo e parto prematuro por trauma cirúrgico. Porém, essa recomendação não possui evidências de boa qualidade, e atualmente a literatura tem demonstrado que pacientes grávidas podem ser submetidas à cirurgia laparoscópica com segurança durante qualquer trimestre, sem apresentar risco aumentado para a mãe e o feto (JONATHAN P, et al., 2017). Chung JC, et al. (2013) também concluíram que a laparoscopia é segura em todos os períodos gestacionais e deveria ser considerada uma alternativa de tratamento padrão à apendicectomia aberta.

Maimaiti A, et al. (2017) concluiu que a videolaparoscopia não aumentou a morbidade e mortalidade, mas apresentou menor tempo de internação e tempo de operação, bem como uma melhor recuperação da função gastrointestinal em comparação aos casos de cirurgia aberta, sendo considerada uma boa abordagem para as pacientes grávidas, sem riscos aumentados.

Já um estudo realizado por Eom JM, et al. (2012), mostrou que a laparoscopia é segura apenas nos dois primeiros trimestres gestacionais, alegando que o posicionamento do útero durante esse período não sofre grandes alterações em relação ao tamanho e, dessa forma, pouco compromete o retorno venoso.

Além disso, no estudo realizado por Walker HGM, et al. (2014), os autores afirmaram que há relatos de complicações maternas associadas à laparoscopia em gestantes, dentre elas infecções de feridas, hemorragia, formação de abscesso, íleo metabólico e eventos tromboembólicos. Porém, não há registro na literatura que revelem índice elevado de mortalidade materna associada à laparoscopia ou de complicações decorrentes dessa técnica em detrimento da cirurgia aberta. Ainda não é possível comparar estatisticamente com a apendicectomia aberta por falta de análises e de estudos publicados relevantes. A conversão de uma laparoscopia para cirurgia aberta foi relatada em apenas um caso neste trabalho.

A literatura atual demonstra que ainda há discordância entre alguns autores, não só quanto à escolha do método mais seguro, como também da idade gestacional segura para a intervenção cirúrgica. Entretanto, diante de uma revisão, conclui-se que a maioria defende a laparoscopia como sendo segura em qualquer período da gravidez (FIGUEIREDO FAS e CORSO CO, 2002).

Os trabalhos divergem também em relação ao uso de antibióticos e agentes tocolíticos, sendo que a maior parte dos autores preconiza o uso de antibioticoterapia no período pré-operatório, com o período máximo de uso por 48 horas, mantendo essa terapêutica apenas nos casos de apêndice perfurado com sepse abdominal. Quanto ao uso de agentes tocolíticos, alguns preconizam utilizá-las rotineiramente. No entanto, como ainda não existem estudos concretos que correlacionam adequadamente a ocorrência de trabalho de parto prematuro durante a apendicectomia com o uso dessas substâncias, a conduta pode variar (DALAQUA M e CORSI PR, et al., 2006).

Um estudo retrospectivo de Eom JM, et al. (2012), foi realizado em gestantes com idade gestacional inferior a 28 semanas mostrou que o tempo médio necessário para realizar videolaparoscopia na gravidez foi significativamente mais curto do que a apendicectomia aberta (27 min vs. $55 \mathrm{~min}, \mathrm{p}$ 1/40,001). Além disso, foi evidenciada e registrada a melhor identificação do apêndice, além de outras causas de abdome agudo que pode ser identificada nesse grupo de gestantes com a utilização da técnica laparoscópica. Os autores relataram ainda que as causas de possíveis complicações foram: necessidade de anestesia geral, possível dano acidental ao útero gravídico, efeitos do aumento da pressão intra-abdominal na circulação útero- 
placentária, uso inadequado de dióxido de carbono e as dificuldades técnicas presentes no terceiro trimestre da gravidez (EOM JM, et al., 2012). Além disso, existem registros de que a ocorrência de diminuição do fluxo sanguíneo uterino (em 40\%) resultou em acidose de curta duração, sem levar a complicações.

O uso de anestesia tem pouca influência em complicações obstétricas e não tem efeitos teratogênicos conhecidos. Lachman E, et al. (1999) afirma que o método aberto de Hasson para criar o pneumoperitônio é o mais indicado para a redução do risco de danos ao útero, pois aumenta a distância do fundo uterino da parede abdominal, facilitando a inserção do trocarte. Considera-se, nesse sentido, as evidências quanto aos benefícios da cirurgia de apendicectomia por videolaparoscopia em relação à cirurgia aberta em gestantes.

Em relação à saúde específica do feto, os resultados ainda não estão bem esclarecidos. Entretanto, os registros encontrados na bibliografia demonstram que potenciais riscos estão relacionados à possibilidade de uma lesão no útero gravídico. Essas lesões podem ser tanto secundárias à inserção do trocarte, como também podem ser decorrentes do aumento da pressão intra-abdominal durante a formação do pneumoperitônio. E tem-se descrito que o aumento da pressão intra-abdominal pode levar a uma redução da perfusão uterina ou até mesmo uma acidose fetal. Em relação aos desfechos fetais, também não foi visualizada associação da técnica laparoscópica com o aumento da taxa de aborto, prematuridade ou APGAR prejudicado no $1^{\circ}$ e $5^{\circ}$ minuto (FERREIRA PEL, et al., 2020).

Mais recentemente, vários autores descreveram resultados bem-sucedidos após apendicectomia laparoscópica após 28 semanas e com dados cada vez mais tranquilizadores, sugerindo que esta abordagem minimamente invasiva pode e deve ser considerada no tratamento da apendicite no terceiro trimestre (SEKAR $\mathrm{H}$, et al., 2019). A técnica de apendicectomia laparoscópica tem a vantagem de uma exploração, diagnóstico e tratamento laparoscópico que não está disponível por meio de uma técnica aberta (NADEEM MA, et al., 2017).

Uma metanálise realizada por Prodomidou A, et al. (2018), a cirurgia videolaparoscópica apresenta diversas vantagens claras para o pós-operatório da gestante em relação à cirurgia aberta, dentre as quais destaca-se o menor tempo de internação hospitalar. Entretanto, esse mesmo estudo ressalta que ainda há carência de estudos comparativos e evidências mais robustas de que a cirurgia aberta, no caso da saúde fetal, seja mais benéfica ou maléfica em comparação à laparoscopia. Nesse sentido, a metanálise demonstrou que o risco de perda fetal verificado na laparoscopia foi de 5,6\% em comparação aos 3,3\% na apendicectomia aberta.

Contudo, estes dados foram tidos como inconclusivos, uma vez que os estudos feitos não levaram em consideração a curva de aprendizado dentro dos procedimentos de apendicectomia laparoscópica, técnica que atualmente apresenta maior difusão. Além disso, deve-se considerar os resultados de complicações da apendicite aguda no pós-operatório em pacientes de diferentes trimestres, como também as admissões na unidade de terapia intensiva neonatal.

\section{CONSIDERAÇÕES FINAIS}

A apendicectomia laparoscópica em gestantes não foi associada a riscos materno-fetais adicionais à própria patologia e pode, desta forma, ser considerada o método de escolha para casos de apendicite aguda durante a gravidez. Além disso, existem vantagens em relação ao método aberto, como o menor tempo de hospitalização, menor prevalência de infecções e dores pós-cirúrgicas, menor ocorrência de íleo paralítico e pouca manipulação uterina. No entanto, é essencial destacar que por ser a cirurgia não-obstétrica mais comum em pacientes gravídicas, os estudos ainda são poucos, visto a importância do assunto, uma vez que compete ao profissional a realização de um diagnóstico clínico, a utilização de poucos exames de imagem e, ainda, uma intervenção cirúrgica de forma precoce.

\section{REFERÊNCIAS}

1. AGGENBACH L, et al. Impact of appendicitis during pregnancy: No delay in acurate diagnosis and treatment. International Journal of Surgery, 2015; 15: 84-89. 
2. BARBER-MILLET S, et al. Actualización en el Manejo del Abdomen Agudo no Obstétrico en la Paciente Gestante. Rev. Cir. Esp., 2016; 94(5): 257-265, 0009-739x.

3. BOUYOU J, et al. Abdominal emergencies during pregnancy. Journal of Visceral Surgery, 2015; 152(6): 105-115.

4. CHAKRABORTY J, et al. Safety of laparoscopic appendicectomy during pregnancy: a systematic review and metaanalysis. ANZ journal of surgery, 2019; 89(11): 1373-1378.

5. CHUNG JC, et al. Clinical outcomes compared between laparoscopic and open appendectomy in pregnant women. Canadian journal of Surgery, 2013; 56(5): 341.

6. DALAQUA M, CORSI PR. Apendicite aguda na gestação. Arquivos Médicos dos Hospitais e da Faculdade de Ciências Médicas da Santa Casa de São Paulo, 2006; 51(1): 4-9.

7. EOM JM, et al. Safety and clinical efficacy of laparoscopic appendectomy for pregnant women with acute appendicitis. Annals of the Academy of Medicine, 2012; 41(2): 82-86.

8. FERREIRA PEL, et al. Desfechos materno-fetais da apendicectomia na gestação: uma comparação entre técnica aberta e laparoscópica. Revista Médica de Minas Gerais, 2020; 30: e-30204.

9. FIGUEIREDO FAS, CORSO CO. Apendicectomia laparoscópica na gestante. Revista do Colégio Brasileiro de Cirurgiões, 2002; 29(5): 284-287.

10. FUENTES-RIVAS A, et al. Appendicitis during pregnancy. Experience at the Hospital General Toluca Dr. Nicolás San Juan. Cir Gen, 2011; 33(4): 218-221.

11. FRANCA NETO AH, et al. Acute appendicitis in pregnancy: literature review. Revista da Associação Médica Brasileira, 2015; 61(2): 170-177.

12. FROUNTZAS M, et al. Is the laparoscopic approach a safe choice for the management of acute appendicitis in pregnant women? A meta-analysis of observational studies. The Annals of The Royal College of Surgeons of England, 2019; 101(4): 235-248.

13. IBIEBELE I, et al. Appendicectomy during pregnancy and the risk of preterm birth: a population data linkage study. Australian and New Zealand Journal of Obstetrics and Gynaecology, 2019; 59(1): 45-53.

14. ISHAQ A, et al. Location of appendix in pregnancy: does it change?. Clin Exp Gastroenterol. 2018; 11: $281-287$.

15. JASCHINSKI T, et al. Laparoscopic versus open surgery for suspected appendicitis. Cochrane Database of Systematic Reviews, 2018; 11.

16. JONATHAN P, et al. SAGES guidelines for the use of laparoscopy during pregnancy. Surgical Endoscopy, 2017; 31(10): 3767-3782.

17. LACHMAN E, et al. Pregnancy and laparoscopic surgery. J Am Assoc Gynecol Laparosc., 1999; 6: 347-351.

18. MAIMAIT A, et al. Laparoscopic appendectomy in pregnancy with acute appendicitis. Surgical Laparoscopy, Endoscopy \& Percutaneous Techniques, 2017; 27(6): 460-464(5).

19. NADEEM MA, et al. Laparoscopic assisted appendicectomy versus laparoscopic appendicectomy. Pakistan Journal of Medical and Health Sciences, 2017; 11(1): 349-351.

20. PERRY ZH, et al. Laparoscopic appendectomy as an initial step in independent laparoscopic surgery by surgical residents. Journal of Laparoendoscopic \& Advanced Surgical Techniques, 2010; 20(5): 447-450.

21. PRODOMIDOU A, et al. Outcomes after open and laparoscopic appendectomy during pregnancy: A meta-analysis. European Journal of Obstetrics \& Gynecology and Reproductive Biology, 2018; 225: 40-50.

22. QUAH GS, et al. Laparoscopic appendicectomy is superior to open surgery for complicated appendicitis. Surgical endoscopy, 2019; 33: 2072-2082.

23. REEDY MB, et al. Laparoscopy during pregnancy: a study of five fetal outcome parameters with use of the Swedish Health Registry. American Journal of Obstetrics and Gynecology, 1997; 177(3): 673-679.

24. WALKER HGM, et al. Laparoscopic appendicectomy in pregnancy: a systematic review of the published evidence. International Journal of Surgery, 2014;12(11): 1235-1241.

25. TINOCO-GONZÁLEZ J, et al. Acute appendicitis during pregnancy: differences in clinical presentation, management, and outcome. Emergências, 2018; 30(4): 261-264.

26. TOWNSEND CM, et al. Sabiston - Tratado de Cirurgia: a base biológica da prática cirúrgica moderna. 19ª ed. Editora Elsevier, 2014; 9788535257670.

27. SEKAR H, et al. Should the laparoscopic approach be the norm for appendicectomy in the third trimester of pregnancy? BMJ Case Reports CP, 2019; 12: e228192. 